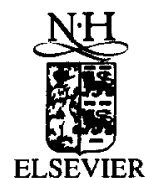

Lingua 108 (1999) 95-117

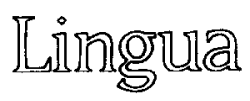

\title{
Using Social Impact Theory to simulate language change
}

\author{
Daniel Nettle* \\ Merton College, Oxford and King's College Research Centre, Cambridge, UK
}

Received 6 May 1998; revised version 22. September 1998

\begin{abstract}
This paper presents a framework for simulating language change in social networks derived from Social Impact Theory. In this framework, the language learner samples the speech of individuals from right across his speech community, though he may weight their input differentially according to their social position. This conceptualisation is argued to be more realistic than that provided by other models. Computer simulations are used to investigate the effects on language change of different social structures and biases in language acquisition. From the results of these simulations, it is argued that the fundamental engine driving language change is the combination of inherent variation in language acquisition and differences between individuals in local social influence. Functional biases attaching to different linguistic variants influence the direction of language change. (C) 1999 Elsevier Science B.V. All rights reserved.
\end{abstract}

Keywords: Language change; Social networks; Actuation problem, Computer simulation

\section{Introduction}

The historical evolution of languages is a complex process, and it would be desirable to have quantitative models of how it works, so that we can make general predictions about its dynamics. Various authors have used models of one part of the process to answer some particular historical or functional question (Keller, 1994; Kirby, 1993, 1995, 1999; Nettle, 1996, 1999), but these models lack generality.

The best-developed frameworks we might adapt for the purpose are the cultural evolution models of Cavalli-Sforza and Feldman (1981) and Boyd and Richerson (1985). There are a number of difficulties in using these models to simulate language

This study was carried out whilst the author was at the King's College Research Centre, Cambridge, as part of the Human Diversity Project. The author thanks Robin Dunbar, Rob Foley, Jim Hurford and an anonymous referee for comments on the manuscript.

* Correspondence to: Merton College, Oxford OX1 4JD, UK. 
change, which are discussed below. In this paper I discuss ways of setting up more realistic alternatives. I present a computer simulation of a model of linguistic change which draws on the theory of social impact from social psychology. I go on to explore the conditions under which linguistic change of the type observed in real communities occurs in the simulated population. In a subsequent paper ('Is the rate of linguistic change constant?', printed immediately following this one in the same issue of this journal), I then use the simulation to investigate the likely effects of such variables as population size on the rate of linguistic change.

For the purposes of evolutionary models, linguistic norms can be seen as an instance of a more general construct, the cultural trait. A cultural trait is any piece of information which is transmitted through a human community by social learning. Languages are evolving populations of linguistic items (Hudson, 1996); these items include words, most obviously, but also all isolatable elements of phonological and grammatical structure. In short, an item is anything which can be individually learned or changed in the history of a language. Linguistic items in this sense are cultural traits. ${ }^{1}$

The best known models of cultural evolution are those of Cavalli-Sforza and Feldman (1981) and Boyd and Richerson (1985). These models take their inspiration directly from population genetics. There are, after all, considerable similarities between the domains of biological and cultural evolution. In biological evolution, a set of items (genes) are transmitted from generation to gencration in a population. Some novelty in this set of items over time is assured because there is a low rate of random mutation. Biological species change because of random fluctuations in the frequencies of different genetic variants, and, more importantly, because of the systematically different success in reproducing which is enjoyed by bearers of different variants (natural selection).

In the cultural, and more specifically linguistic, domains, items are also passed from generation to generation. There is also, it seems, a source of variation which is constantly providing new slightly different items. This source is usually held to be the imperfect learning of languages. The idea that learning is imperfect dates back at least to Henry Sweet:

"If languages were learnt perfectly by the children of each generation, then languages would not change ... The changes in languages are simply slight mistakes, which in the course of the generations completely alter the character of the language." (Sweet, quoted in Aitchison, 1991: 165)

Since then, despite a general lack of direct evidence that language learning is imperfect, this mechanism has repeatedly been put forward as the fundamental source of historical change in language (King, 1969; Andersen, 1978). Imperfect learning is not the only candidate source of variation, though. Other linguistic tradi-

\footnotetext{
1 This conceptualisation of language does not depend on a claim that the items which make up a language are statistically or functionally independent. Languages are systems, in which individual elements depend upon each other both for their contrastive functioning and their diachronic persistence. This does not however undermine the usefulness of treating any element that can in principle change independently as a separate item (see Nettle, 1999 for a discussion).
} 
tions have stressed the way in which the vagaries of linguistic performance in real time lead to variation in speech output, which can also serve as input to the evolutionary process (Luidtke, 1986; Lindblom et al., 1995). Clearly, the type and degree of variation produced by these two mechanisms is very different. Variants due to imperfect learning will normally stay with an individual for his entire lifetime and be produced on every occasion. Performance variants may be one-offs, which would make them much less likely to be adopted into the language, though they may recur in the speech of several individuals or on several occasions if production constraints favour them. Indeed, one of the central insights of the phonetic approach to sound change is that the slips which the articulators or ears are most likely to make are those which are most likely to be adopted into a language (Lindblom et al., 1995). The source of variation in cultural evolution models is of the imperfect-learning type, and for convenience I will adopt the same strategy here, though this is an area which requires further investigation.

In biological evolution, an individual inherits genes from its two parents, with a basically even probability of getting the variant of the father or that of the mother. This is where the similarity between biological and linguistic evolution ends, as the inheritance of linguistic norms is quite different. The process of language acquisition is quite a long one, during which the learner samples not just his parents, but a wide range of sources. In small communities, he may even sample all other speakers before the period of plasticity during which language acquisition occurs is over. In larger societies, learners are certainly exposed to a wide representative sample from across the speech community. The learner somehow extracts his own linguistic behaviour from this mass of often heterogeneous data. Furthermore, the different individuals in the sample are certainly not given equal weight; their influence rather depends on their social distance from their learner and other social factors. Peer groups and other role models seem to be particularly important. The items he ends up with will not be those of any one model, but will in all probability represent a composite of different inputs. Exactly what kind of algorithm he uses to extract this composite from a large and often conflicting data set is the problem I wish to address in this paper.

In the cultural evolution models of Boyd and Richerson (1985), the learner has a fixed number of 'cultural parents' from whom he obtains his cultural repertoire. This number can be varied, but the mathematics becomes intractable as the number of parents becomes large, and for this reason, many of the results using this framework have been derived for the case where the number of parents is just two, as it is in biological inheritance. This is clearly inappropriate for acquisition of linguistic items.

Cultural evolution models have a number of other attributes which make them unsuitable for the present problem. They are 'mean field' models; that is to say, the assume an infinite population which has no particular spatial or internal structure. Thus they can be used to give information about the expected outcome of a process in the limit when the population is large. They tell us much less about the dynamic fluctuations and variations which will occur in a population which is finite, and socially and spatially structured, which of course all speech communities are. For related reasons, Boyd and Richerson's models provide no basis for computing the 
weighting of different cultural parents to different learners, since they employ no concept of social structure. In this paper, then, I outline an alternative model derived from social psychology which incorporates a notion of social structure.

The disanalogy between cultural and biological evolution means that there is a huge problem for the theory of language change which does not exist in population genetics. I call this the threshold problem, and one of the key questions for the present simulation will be that of how the threshold problem is overcome in language change. The threshold problem may be outlined as followed.

\subsection{The threshold problem}

When a new genetic mutation first arises, it is by definition extremely rare. In fact at some point it is found in only one individual. When that individual reproduces, there is a good chance ( $100 \%$ for asexual organisms, normally $50 \%$ for sexual ones) that this mutant will be passed on. If the parent has several offspring, the mutant can thus increase its frequency. As long as the mutant is not severely deleterious or actually lethal, then, there is a good chance that its population frequency will become appreciable. Indeed, a fairly high rate of genetic change can be expected just by chance changes in gene frequencies, or drift, without even considering the role of natural selection (Kimura, 1983). Cultural evolution models often make a similar assumption for cultural traits. When a rare mutant trait arises in an individual, it has a good chance of being passed on to that individual's cultural 'offspring' (the exact figure depends on the particular model and the number of cultural parents used; it can be as high as $50 \%$ ).

In reality, however, the situation is quite different. A language learner samples across a fairly large number of people in the generations above him and in his peer group. He derives his linguistic repertoire by some algorithm from this sample. Now any mutations which have arisen in the previous generation will at the time the next generation is learning be extremely rare. In the sample the learner is exposed to, they will certainly be in the minority. All plausible learning algorithms lead, other things being equal, to the adoption of the most common variant in the sample for a given item, which will never be the new mutant. Thus, even where there are many new variants arising through imperfect learning, no change can be expected in language as the next generation will simply discount the rare aberrant forms in favour of the more common ones. New mutants can only become fixed in a language if they can pass a threshold of frequency which in the early stages they never have.

In existing computer simulations of language change, such as those described in Keller (1994) and Kirby (1993), individuals acquire their norms from just a few immediate neighbours in a spatially organised population. This masks the threshold problem, so in those models rare variants successfully increase their frequency. We know this is unrealistic, though, since real language learners have dozens or hundreds of models rather than four or five.

The threshold problem is a major difficulty for the theory of language change which has been recognised for at least 70 years. As Sapir puts it: 
"The explanation of primary dialectic differences is still to seek ... If all the individual variations within a dialect are being constantly levelled out to the dialect norm ... why should we have dialect differences at all?" Ought not the individual variations of each locality, even in the absence of intercourse between them, to cancel out to the same accepted speech average? (Sapir, 1921/1970: 149-150)

Keller recognises basically the same problem, which he calls Humboldt's problem; if the learner adopts whatever norm is most common around him, then the result after a few generations is always "homogeneity if the starting point is heterogeneous and stasis if the starting point is homogeneous" (Keller, 1994: 99).

Despite this recognition of the problem, it is not brought up in most discussions of language change, where the bridge between the origins of new variants and their transmission to new speakers seems to be regarded as unproblematic. The exception to this generalisation is the sociolinguistic literature, to which I return below.

We have, then, a conceptual problem. In real languages rare variants get adopted and spread through entire communities in a rising $S$-shaped curve. Even given the existence of sources of variation, it is not entirely clear how this can happen. It might be the case that chance alone, coupled with a sufficiently high rate of variation due to imperfect learning, is sufficient to occasionally overcome the threshold problem and allow language to change. Whether this is likely to be the case is one of the questions to be asked of our model. On the other hand, the solution may well lie in the fact that the learner does weight all the sources to which he is exposed equally but uses a biased learning strategy. If the learner were at least sometimes biased towards new variants for some reason or other, then those variants would have a chance of overcoming the threshold of rarity.

There are two possible sources of such a bias. One is social; the learner may favour the speech of some individuals more than others, and so, if socially influential people are from time to time the bearers of new variants, transmit those variants. The other is linguistic or functional; certain linguistic variants may have some functional attribute which makes them easy to acquire or use which favours their adoption over their competitor variants. I shall incorporate biases of each type into the computer simulation to examine their effect on the dynamics of language change. First, however I discuss the rationale for each of them in turn. ${ }^{2}$

\subsection{Social selection}

One of the central insights of sociolinguistics is that learners of language do not simply pick up the most common norms going on around them. Rather, they are seen as active discriminators who aspire to membership of particular social groups and make themselves sound as similar as possible to the members of those groups. LePage (1968:192) lays out this approach to language acquisition:

"Each individual creates the systems for his verbal behaviour so that they shall resemble those of the group or groups with which from time to time he may wish to be identified, to the extent that

2 Social and functional selection are referred to in Boyd and Richerson (1985) as 'indirect bias' and 'direct bias'. The threshold problem is partly foreshadowed in their discussion of 'conformist' bias. 
(a) he can identify the groups,

(b) he has the opportunity and ability to observe and analyse their behavioural systems,

(c) his motivation is sufficiently strong to impel him to choose, and to adapt his behaviour accordingly,

(d) he is still able to adapt his behaviour."

Numerous empirical studies have shown that LePage's formulation is in essence correct. Speakers do not simply copy the most frequent items around them; they rather model themselves on those with whom they wish to associate and identify, and with whom their aspirations are bound up (Labov, 1963; Milroy, 1980; for a review of other studies see Chambers, 1995; Hudson, 1996). This has implications for the way we model language change. Rather than assuming that all speech input is equally weighted in the learning process, we should take account of the fact that different individuals are influential to different degrees, depending upon their place in the social structure. Thus, a rare variant might overcome the majority norm if the individual(s) using it were especially influential. Inter-individual differences in influence are well-understood in social psychology, and are a central component of social impact theory, on which I draw in the simulation reported in this paper.

\subsection{Functional selection}

Under social selection, it was assumed that learners are biased towards particular people. Under functional selection, we consider the possibility that learners may have biases towards particular linguistic items. For example if one variant of a linguistic item were easier to learn than another - if it involved, for example, a simpler rule, or a more audible phonetic discrimination - then a learner's acquisition would be weighted in its favour. Arguments that perceptual or cognitive biases of this kind exist have been frequently put forward in the literature on typological regularities across languages and in 'functionalist' approaches to language in general (see e.g. Cutler et al., 1985; Tomlin, 1986; Hawkins, 1993; Kirby, 1999).

Functional selection then, is another possible mechanism which might allow the threshold problem for new variants to be overcome, and linguistic change to take place. Which of these two biases, functional or social, if any, is important is an empirical question whose answer is some way off. The model and computer simulation outlined below may, however, help to give some indications.

\section{Social Impact Theory}

The simulation reported here draws on an adapted version of Social Impact Theory (Latané, 1981). Social Impact Theory was developed as a metatheoretical framework for modelling situations where beliefs, attributes or behaviours of an individual are influenced by those of others around him. It has been applied to a large number of social psychological situations by Latané and coworkers (see e.g. Latané and Nida, 1981; Latané and Wolf, 1981). It has also been adapted to serve as the basis 
for computer simulations of attitude change in social groups (Nowak et al., 1990). The current simulation draws heavily on this last paper.

Social Impact Theory (especially as adapted by Nowak et al. 1990) seems appropriate for the simulation of language learning, since it postulates a situation in which the traits acquired by an individual are related to those held by the social group around him by a complex function which allows for the differential influence of different sources, differential proximity of different sources within the social structure, and of course the number of sources in the group having different traits. I have freely adapted the formulation given in Nowak et al., (1990) as seems to me appropriate for the linguistic case; the reader is referred to that paper and to Latane (1981) for the original formulation.

Let us consider the case of a linguistic item where there are two competing variants in use in a population, $p$ and $q$. The learner is exposed to speakers using each variant in his social group. However, in arriving at the one he finally adopts he will be influenced by the number of speakers having each variant, who those speakers are, and possibly by a bias in acquisition towards one variant or the other. We can attempt to formalise this situation by saying that the speaker will adopt the variant which has greatest impact upon him. The impact $\hat{\imath}$ of some variant $p$ can be defined by the following formula.

(1) $\hat{i}_{p}=\mathrm{b}_{p} f\left(\mathrm{~S}_{p} \mathrm{D}_{p} \mathrm{~N}_{p}\right)$

Where $\mathrm{S}_{p}$ is the status of the individuals using variant $p, \mathrm{D}_{p}$ is their social distance from the learner, $\mathrm{N}_{p}$ is their number, $\mathrm{b}_{p}$ is a constant used to represent any acquisitional bias towards or against $p$, and $f$ is some function to be specified. I have used the concept of status, $S$, to capture the observation that there are absolute differences in influence or social standing between individuals in any community. This allows for the incorporation of social selection, as described above, into the model. Sociolinguists would be quick to observe that learners often aim not for the variable with the highest overall social status, but that which produces the greatest social solidarity. In this context, however, I intend status to be interpreted as a more local construct, indicating whatever it is that makes some individuals more socially attractive or influential than others within a particular group. D, the variable of social distance, allows for the fact that within the social structure of any group, some individuals are more closer than others, and are therefore likely to have greater impact on them. The inclusion of $\mathrm{N}$ allows for the fact that other things being equal, variants that are heard more often will be more likely to be adopted. Finally, $\mathrm{b}$ can be altered to allow for the possibility of biases in learning of the type described under functional selection, above.

The impact of the competing variant $q$ is simply:

(2) $\hat{\imath}_{q}=\mathrm{b}_{\mathrm{q}} f\left(\mathrm{~S}_{q} \mathrm{D}_{q} \mathrm{~N}_{q}\right)$

We assume that the learner will adopt $p$ if $\hat{\imath}_{p}>\hat{\imath}_{q}$ and $q$ if $\hat{\imath}_{q}>\hat{\imath}_{p}$. Note that equations (1) and (2) reduce to the simple strategy of 'adopt the most common variant' in the 
case where all individuals have equal status and social distance from the learner, and $b_{p}$ and $b_{q}$ are equal (that is, there are no functional biases). Note also that the Social Impact model reduces to a standard two-parent cultural evolution model if just two source individuals are considered and if they are given equal status and distance from the learner.

Social Impact Theory further specifies the nature of the impact function. Specifically:

(3) $\hat{\imath}_{p}=\mathrm{b}_{p} \mathrm{~N}_{p}{ }^{\mathrm{a}}\left[\Sigma\left(\mathrm{s}_{\mathrm{i}} / \mathrm{d}_{\mathrm{i}}{ }^{2}\right) / \mathrm{N}_{p}\right]$

In (3), $\mathrm{s}_{\mathrm{i}}$ is the status of the $i$ th individual with variant $p$, and $\mathrm{d}_{\mathrm{i}}$ is the social distance of the $i$ th individual with variant $p$ from the learner. The symbol $a$ represents an exponent of $\mathrm{N}_{p}$, which must be chosen in the specification of the model, and to which we return below. The net impact of a particular individual $i$ with variant $p$ on the learner is $\mathrm{s}_{\mathrm{i}} / \mathrm{d}_{\mathrm{i}}{ }^{2}$, the status of $i$ divided by square of the distance from the learner. The distance $d_{i}$ is always squared in Social Impact Theory, apparently by analogy with the laws of gravitation, where effects decrease with the square of distance, but there is no absolute reason why this should be the case, and in the simulations I will experiment with raising distance to higher powers. I also experiment with raising status to a higher power. The summation $\Sigma\left(\mathrm{s}_{\mathrm{i}} / \mathrm{d}_{\mathrm{i}}{ }^{2}\right)$ is the sum of the impacts of all the individuals with $p$, and $\Sigma\left(\mathrm{s}_{\mathrm{j}} / \mathrm{d}_{\mathrm{i}}{ }^{2}\right) / \mathrm{N}_{p}$ is therefore the average impact of an individual with variant $p$ on the learner. To obtain the total impact of variant $p$, this last quantity must be multiplied by the number of individuals with $p$. However, this multiplier is not simply $\mathrm{N}_{p}$ but rather $\mathrm{N}_{p}$ to the power of $a$.

The inclusion of the constant $a$ in Social Impact Models in general is to allow for the fact that the persuasiveness of a belief does not rise linearly with the number of people holding it. If this were generally true, then total agreement with the majority view would be the expected outcome of most social processes. In fact this is not the case, and most groups harbour a diversity of opinions on most matters. Social Impact Theory applied to matters of public opinion therefore uses an a coefficient of less than 1 - usually around 0.5 - to allow for the fact that beyond a certain point increasing the number of people who hold some belief does not lead to proportional increases in the probability that some other individual will convert to that belief. In the linguistic case, I will experiment with various values of $a$, but ultimately argue that a higher value is appropriate. This is because language is a domain around which communities tend to reach consensus more than they do in, for example, fashion or choice of political party. It is true that there is a great deal of stable linguistic variation within complex societies, but it seems that that reflects the fragmentation of those societies into subunits with partial social boundaries around them, and abrupt increases in social distance between them, rather than a failure to reach consensus within cohesive social groups.

Equation (3), then, gives the net impact of variant $p$ on a language learner. It has a counterpart equation (4) which does the same for the variant $q$.

$$
\hat{i}_{q}=\mathbf{b}_{q} \mathrm{~N}_{q}^{\mathrm{a}}\left[\Sigma\left(\mathrm{s}_{\mathrm{i}} / \mathrm{d}_{\mathrm{i}}^{2}\right) / \mathrm{N}_{q}\right]
$$


The variant adopted will be the one whose net impact is greatest. (Note that we only consider the case of a two-way choice between variants here. Multiple variants could easily be accommodated within the theory as long as the different variants could be enumerated.) These, then, are the fundamental equations governing the Social Impact simulation. So far, it is a static description of a single learner. It becomes an interesting approach to language change only when we set up a dynamic population of speakers who live and die within a defined social structure, learn some language using the equations, and serve as models to subsequent learners. That is the purpose of the computer simulations described in the next sections.

\section{The simulation}

A computer simulation is never a realistic replication of the situation it aims to elucidate. Thus it can very rarely be used to make precise empirical predictions about real world processes. One might therefore ask what the point is of doing it. The answer is that though it is not a total replication of a real world situation, it attempts to take the main relationships of a real world situation and explore their general effects across a range of conditions. This is useful because complex iterated processes of the kind found in social evolution can have unlooked-for, complex dynamics which cannot be predicted by simple thought or deduction. A simulation, however rudimentary, is thus an improvement over a merely verbal argument, in deciding what general conditions must obtain for languages to evolve in the way that they do. It is in this spirit that I would like the following simulation to be taken; highly simplistic, but, I hope, interesting as a way of exploring issues surrounding language change which is more systematic and better supported than mere speculation.

Critics of computer simulations often complain that the author has simply built into the model whatever he desires, so when that desideratum results it is not interesting. This is a misunderstanding of what simulations such as this one are for. I will indeed tweak this system until it produces results that resemble real linguistic change in some ways. The interest lies therefore not in what it can be made to do, but rather in what assumptions and initial conditions have to be included to make it behave in the desired way. These assumptions and conditions may give us some general insights into the conditions of real language change.

The simulation takes the maximally simple case, of two competing variants, $p$ and $q$, of the same grammatical item. A population of 400 individuals is set up, each of whom learns one or the other variant using the algorithm described by equations (3) and (4). The evolution of the population norm is then tracked. In this section I describe and explore a basic version of the simulation in which there are no functional biases (that is $b_{p}$ and $b_{q}$ are equal to 1 ), and all individuals have the same status (that is, there is no social selection). The basic structure of the simulation will be the same in subsequent sections where social and functional selection are added.

The 400 individuals are arrayed on a 20 by 20 grid. This is not necessarily meant to represent a spatial structure. Rather it is a representation of a social structure; 
those closer on the grid are assumed to be socially closer to each other than those who are more distant.

The individuals pass through five lifestages before death. The rows of the grid have initial age distributions as follows:

\section{(5) 54321123455432112345 \\ 54321123455432112345 \\ 54321123455432112345}

This structure was chosen because it incorporates both familial and horizontal social ties. That is, an individual is closest to others older and younger than him across the horizontal of the grid, who might be thought of as parents and siblings, and closest to his own peer group, or part of it, up and down the vertical of the grid. Thus a whole cohort along the vertical of the grid goes through language acquisition at the same time. At the end of each lifestage, all ages are increased by one, and those of age five die and their places are taken by new individuals of age 1 . The grid is toroidal; that is, any individual in the leftmost row is adjacent to his counterpart in the rightmost row, and similarly the top row is joined to the bottom one. The use of a toroidal space is to eliminate 'edge' effects. If the space were a simple plane, the individuals at the edges would have fewer neighbours than the others and would thus behave differently. Such differences might be of interest in other contexts, such as in the explanation of the differences found in dialect geography between core and peripheral areas (Andersen, 1988), but for present purposes it is desirable to remove them.

During the first lifestage, individuals learn from those around them by evaluating equations (3) and (4) and adopting $p$ or $q$ accordingly. The equations mean that they are influenced by all the other individuals, except for those of age 1, who are still learning and have no impact as yet. The influence is however not equal but declines with distance from the learner.

During the second lifestage, which I envisage as adolescence, individuals re-evaluate and may still change their variant. However, during their second lifestage they also influence others of their age and below. These adolescent cohorts are very important as they are adjacent to the new learners and also inclined to innovate and change, as we shall see. Learning update is sequential along the rows and down the grid.

During subsequent lifestages, no further learning or change occurs. That is, I assume that language acquisition takes place during a critical period ending at the end of adolescence (Hurford, 1991).

Variation stems from imperfect learning. In both the first and the second lifestage, individuals may with a certain probability, usually 0.05 , adopt the contrary variant to that indicated by learning. We can call this a mutation rate of $5 \%$. If mutation happens during the first lifestage, there is often a fairly high chance that the error will be corrected by the relearning in the next lifestage. If it happens in the second lifestage, it will stay with the individual for life. 
The simulation is generally initiated with a homogeneous population using $p$. Various measures of the subsequent evolution of the population are then tracked as the generations pass. The 'norm' is simply which of the variants is most common. The 'homogeneity' is the proportion of individuals who have the norm. 'Changes' is the number of times the norm has changed in a specified time period. ' $\% \mathrm{p}$ ' and ' $\% \mathrm{q}$ ' are the percentages of the population having $p$ and $q$ respectively.

\subsection{Basic results}

Let us consider the basic case where there are no functional biases, all individuals have the same status, and $a=1$. The results when the simulation is run for 200 generations can be appreciated by examining Figs. 1 and 2. Fig. 1 shows the percentage of individuals having $p$ each generation. This percentage never reaches $100 \%$ since a few $q$ s arise each generation because of imperfect learning, but neither can $q$ ever spread because of the overwhelming influence of the $p$ majority. Fig. 2 shows the grid after the 100th run; the few $q$ individuals are scattered around, and there is no sign of $q$ taking hold as the norm in any part of the space.

This is a graphic illustration of the threshold problem; $q$ arises, actually fairly frequently, through random imperfect learning, but it cannot never take hold more generally because of the overwhelming influence of the $p$ majority. There are several possible modifications which could be made to the simulation in order to overcome the threshold problem. The two discussed above are the additions of social and functional selection respectively. Before turning to those, however, we will examine the

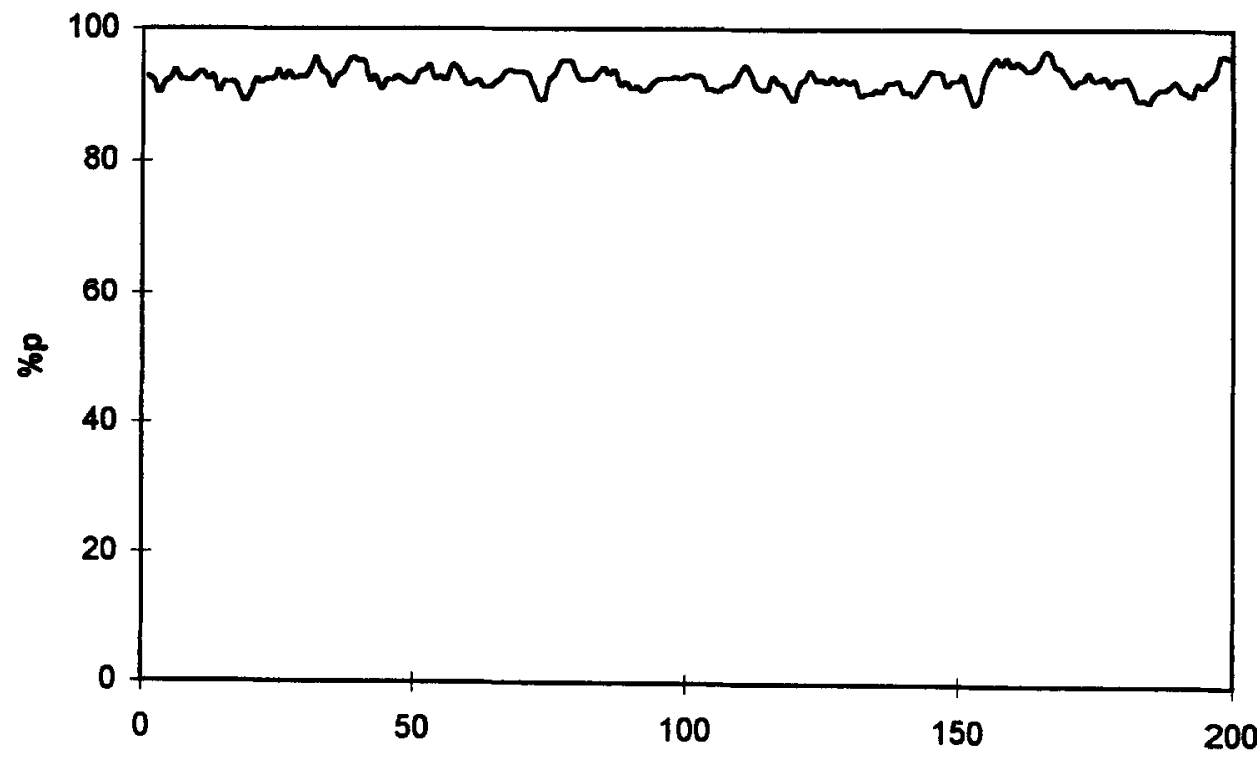

Fig. 1. The percentage of individuals having $p$ over 200 lifestages in the basic simulation. 


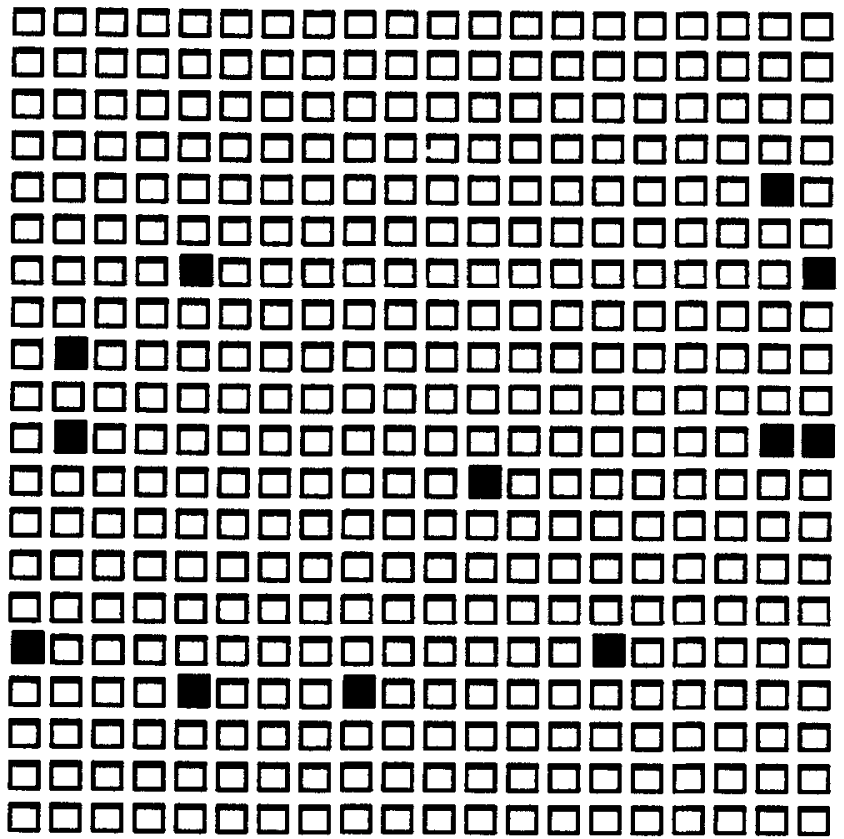

Fig. 2. The output of the simulation after 100 lifestages. Each square represents an individual; open if the individual has variant $p$, filled if he has $q$.

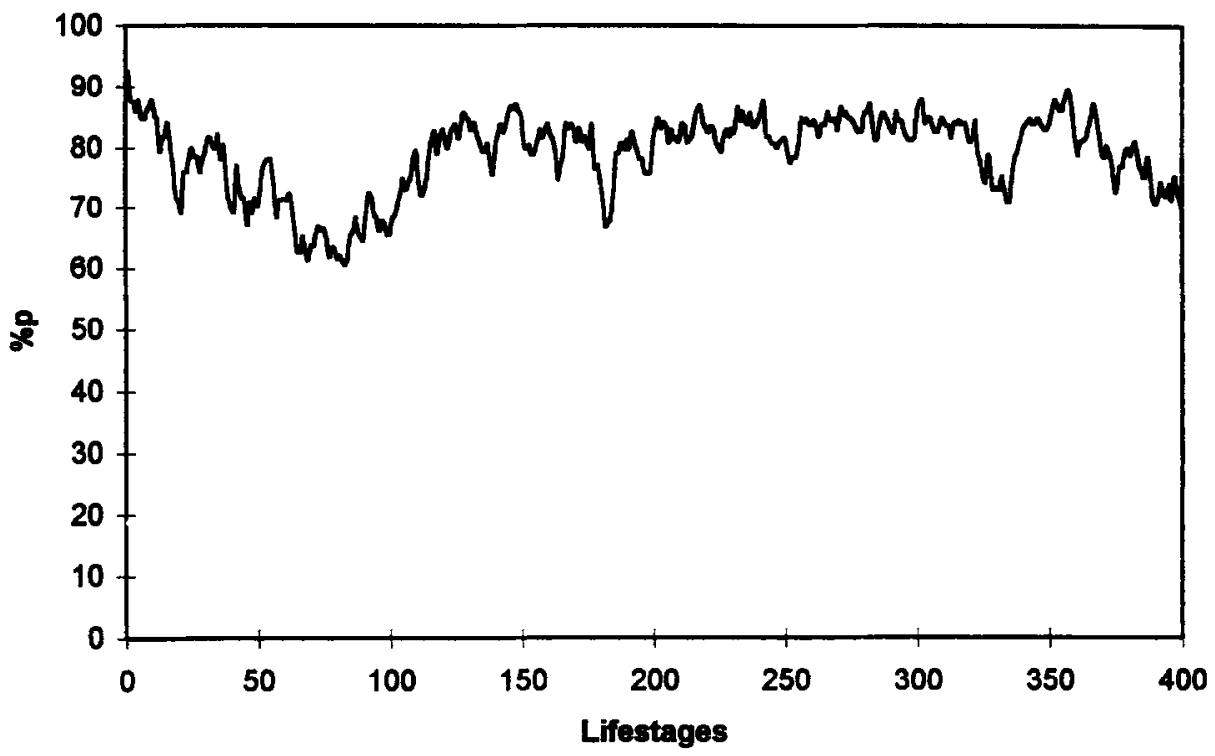

Fig. 3. The percentage of individuals having $p$ over 400 lifestages in the basic simulation, with the exponent $a$ set to 0.5 . 
effects of manipulating three other parameters within the basic simulation. These are the constant a, which governs the effect of majority consensus on impact; the rate of mutation (imperfect learning); and the weighting of social distance.

\subsection{Varying the constant a}

Recall that lowering the constant $a$ to less than one means that the total impact of $p$ rises less than linearly with increasing number of individuals using $p$. This in effect reduces the importance of large numerical majorities in the learning process. If we lower $a$ to 0.5 , then the rare variant $q$ is indeed able to increase its frequency eventually, as Fig. 3 shows. As Fig. 4 illustrates, this is because it becomes established in one part of the space and then is impossible to dislodge. The eventual equilibrium state of the simulation with $a=0.5$ is always half $p$ and half $q$, with the two variants occupying different regions of the space. This is also true for any value of a lower than 0.5 . Values of $a$ between 0.5 and 1 produce the same result as $a=1$. Thus it seems that there are just two equilibria for the system; basically all $p$, or half $p$ and half $q$, with an abrupt transition between them as the value of $a$ changes.

Setting the constant $a$ to less than one, then, is one way in which change may be actuated within the simulation, and, indeed, in other Social Impact Theory models,

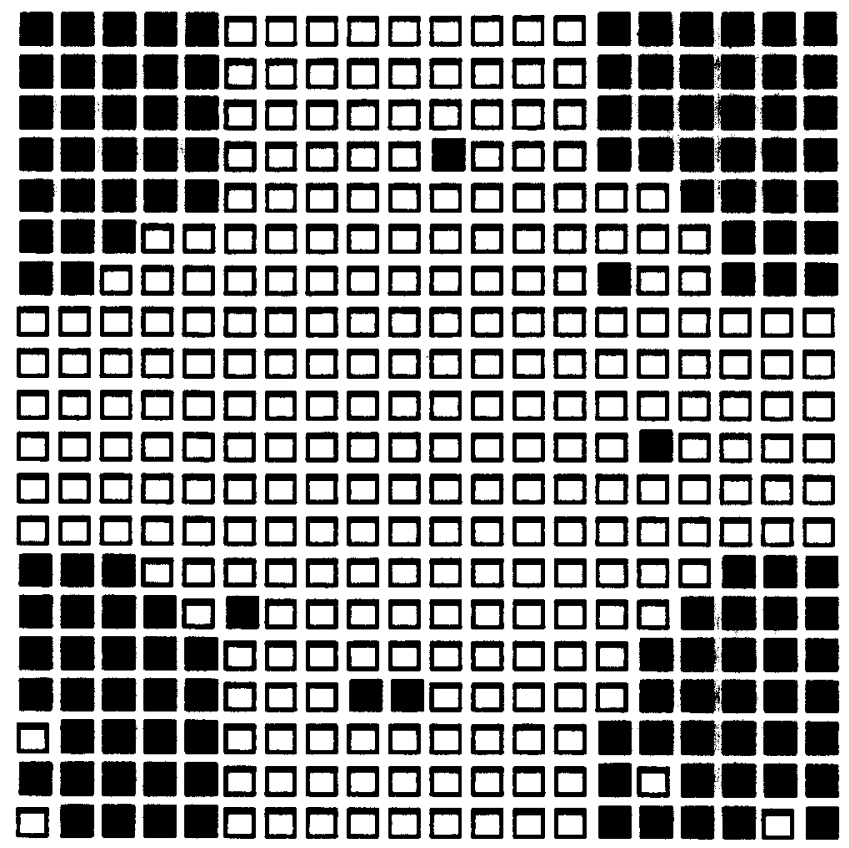

Fig. 4. The output of the simulation after 100 lifestages with the exponent $a$ set to 0.5 . Each square represents an individual; open if the individual has variant $p$, filled if he has $q$. Note that due to the toroidal shape of the space, the area of filled squares is one continuous region. 
such as that of public opinion, values of $a$ of around 0.5 are normal. However, this is not a very satisfactory solution for a linguistic model. An $a$ value of 0.5 allows for the persistence of a diversity of traits within a group; indeed, it was chosen in Social Impact Theory expressly because it predicted the stability of heterogencous opinions within cohesive groups. The linguistic situation is somewhat different, however. There are many linguistic variants which remain rare or marginal; when they do pass a threshold, however, they can spread quickly through the entire community, and speech communities generally achieve a kind of consensus on linguistic norms which is lacking in say, public opinion. ${ }^{3}$ The situation observed with $a=0.5$, that of a population at equilibrium split equally between two dialects, is not how we would expect a real speech community to be. Of course, there are dialectal divisions in real societies, but these are along partial social boundaries. The simulation population is a single integrated group with no social discontinuities. A higher $a$ value than 0.5 is therefore desirable to achieve community consensus. On the other hand, a value of 1 gives the initially common variant such a massive advantage that it can be displaced under almost no circumstances. The value I have chosen for subsequent simulations is therefore 0.8 , which gives similar behaviour to $a=1$ in the basic simulation, but allows rare variants to spread occasionally and thus permits the simulation to exhibit more interesting behaviour in the more complex cases to be described below.

\subsection{Varying the mutation rate}

In the simulations described so far, the variant $q$ never overcomes the threshold of rarity to become the norm. In real language change, however, this happens not infrequently. One reason for the discrepancy between the simulation and reality may be that the rate of imperfect learning, and hence of mutation to the rare variant, is

Table 1

The mean proportion of individuals having the majority variant, and (bracketted) the number of changes of norm, over 200 lifestages for different values of the mutation rate and different exponents of social distance

\begin{tabular}{lll}
\hline Mutation rate & Distance power 2 & Distance power 4 \\
\hline $5 \%$ & 0.96 & 0.69 \\
& $(0)$ & $(45)$ \\
$10 \%$ & 0.92 & 0.57 \\
& $(0)$ & $(68)$ \\
$20 \%$ & 0.85 & 0.54 \\
& $(0)$ & $(78)$ \\
\hline
\end{tabular}

3 There is no contradiction between this statement and the observation that certain classes of linguistic change take centuries to complete. Examples include the breakdown of the strong verb system in English, or indeed the great vowel shift. The slow diffusion in these cases is from one item to another within the language, not from one individual to another within the community. 
higher than the one in twenty reported here. This seems unlikely; one in twenty is already orders of magnitude higher than the rates of mutation in genetics and seems very high as a probability of a learning algorithm missing its target. The effect of higher rates is nonetheless worth investigating.

The first column of Table 1 shows the mean HOMOGENEITY (the proportion of individuals having the common variant) over 200 lifestages with the probability of imperfect learning set at $0.05,0.1$ and 0.2 . Obviously, increasing the rate of imperfect learning decreases the homogeneity, because more random innovations are occurring, but by itself it never leads to changes in the overall norm. The rate of imperfect learning was not increased further than $20 \%$, which is already extremely high. Related simulations in Nettle (1996) indicated that imperfect learning alone could lead to changing norms only when the rate was increased well above $25 \%$, which is implausible and reduces learning to little better than chance.

Increasing the rate of mutation, then, does not of itself seem capable of producing orderly linguistic change. We will instead examine the effect of weighting the variable of social distance.

\subsection{Varying the weighting of social distance}

In the simulations described so far, the net impact of an individual $i$ on a learner decreases with the square of the distance from $i$ to the learner. One could increase the effect of distance, having, for example, the impact decline with the fourth power of the distance. The effect of this would be to make learners more parochial; that is,

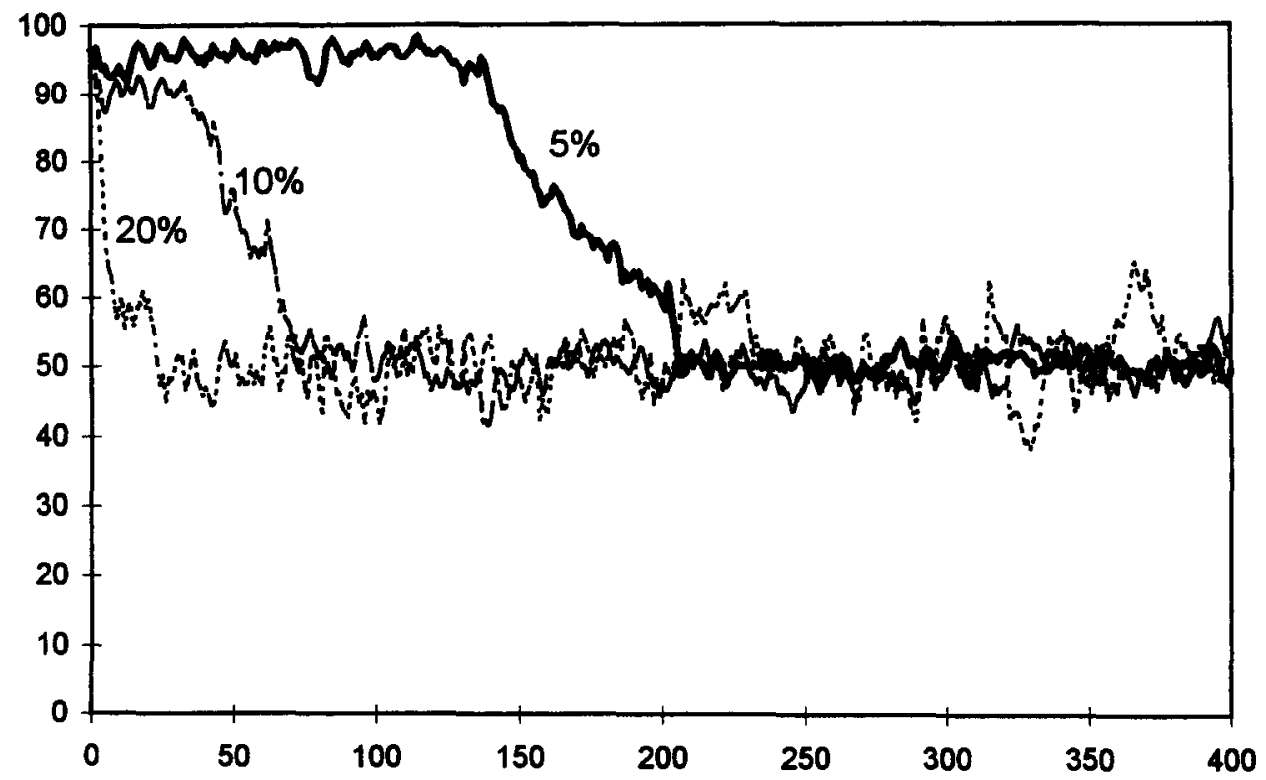

Fig. 5. The percentage of individuals having $p$ over 400 lifestages, with the variable of distance raised to the power four and three different values of the mutation rate $(5 \%, 10 \%$ and $20 \%)$. 
instead of an individual twice as far away having one quarter of the impact of a nearer one, he would have one sixteenth of the impact. Similarly, an individual ten links away in social space would have one hundredth of the impact of one next door if distance were squared, and one ten thousandth of the impact if distance were raised to the power four.

The results of changing the exponent of distance are given in Table 1. With $a=0.8$, raising distance to the power 4 pushes the simulation to the same $50 / 50 p$ and $q$ equilibrium as was observed by lowering the value of a. The very large numbers of changes of norm are observed because the simulation is oscillating around the 50/50 level. How fast this equilibrium is reached depends on the mutation rate (Fig. 5). This finding is not surprising; by making speakers more parochial, one increases the likelihood of their forming separate dialect patches, and the faster the rate of mutation, the faster this will happen.

Raising the effect of distance in this way still does not produce a situation in which a rare variant can spread and replace a previously dominant one population wide. Besides, the extreme parochialism implied by raising distance to the power four almost reduces the Social Impact model to a cultural evolution model with a small number of cultural parents, since the impact of non-adjacent individuals is reduced to a negligible level. This is unrealistic and is to be avoided. Furthermore, there is some independent empirical evidence that the effect of geographical distance on the diffusion of cultural traits does indeed follow a quadratic function (Hägerstrand, 1965). For these reasons, distance has been left squared for subsequent simulations.

The lesson of the basic simulation seems to be that with no status differentials between people and no functional biases, it is extremely hard to see how rare variants ever overcome the threshold problem and spread. Under extreme conditions such as high parochialism and mutation rates they can achieve local persistence, but the result in these cases is odd heterogeneous equilibria. Nothing akin to communitywide language change is observed. This may be because in real linguistic evolution social influence differentials and/or functional biases are of key importance. In the next two sections, then, I add each of these complications to the simulation.

\section{Social selection}

The idea of social selection is that different individuals have different levels of social influence within a community, and that learners model themselves differentially on different sources. We represent social influence by the status variable already discussed.

The simplest way of incorporating social selection is to give all individuals, from adolescence, a randomly assigned value of status. This value is not related to whether the individual has learned $p$ or $q$; we thus assume it reflects the individual's non-linguistic attributes or position. We have to consider what would be the optimal population distribution of status. Many human attributes, such as height and weight, are normally distributed. However, attributes of relative social position are never dis- 
tributed like this. Across all societies, for example, the distribution of wealth is a Poisson curve. That is, there is a large cluster around the median, and then a long tail to the right in which a few immensely rich individuals reside. This means that the mean and the median are quite different, because the mean is pulled up by a few vastly wealthy individuals (see, for example, Wolff, 1998).

It is a reasonable supposition that the distribution of social status is more like that of wealth than that of height. There are a few individuals who are immensely influential, and a great bulk who are around the average. Why such distributions should be typical of cultural success traits in human societies is another question. In the wealth case, it is because people in the top tail of the distribution can use their wealth to make more wealth, thus pulling them even further away from the median. The same must be true of social influence processes more generally. People who are influential can organise things so as to further enhance their own influence, and are also looked to simply because they are already well-known (which is why best-selling books remain best-sellers, and widely cited papers become more widely cited; for an economic perspective on such distributions, see Bikhchandani et al., 1998).

I therefore submit that the distribution of the status variable should be of this Poisson type, with a few individuals being much wealthier than the bulk of the rest. The most computationally convenient way to do this is to assign a status value of 1 to 20 with equal probability to each individual, thus giving a uniform distribution, and then to square that value, giving a range of $1-400$ with 20 possible values of status and most individuals in the lower half of the range.

When status is incorporated into the simulation in this way, with a mutation rate of $5 \%$ and $a=0.8$, the results are only subtly different (Fig. 6). Small pockets of the rare variant form around high status individuals who might happen to have it. However, these pockets never become large enough for the rare variant to achieve critical mass and spread to the whole population, not least because the influential individual at the centre of the pocket dies out after 4 lifestages.

For sustained change to occur, there must be even greater influence differentials between speakers. To implement this, the distribution of status was made even more skewed. An initial status of 1-20 was assigned as before. The, with a probability of $1 / 40$, this value was changed to 100 , creating a small set of hyperinfluential individuals. The status values were then squared as before.

The results of running the simulation with this modification are shown by the second line Fig. 6. Now, very occasionally, when a hyperinfluential individual happens to have the rare variant, it quickly spreads in his immediate neighbourhood. If that spread is fast enough, the rare variant then attains critical mass and replaces the dominant in a rapidly rising curve similar to that characteristic of real linguistic change. The population then stabilises at near homogeneity with the previously rare variant as the norm, and remains in that state for a long equilibrium period until another change is triggered. The mean homogeneity of the population is high $(0.86$ over 400 lifestages), and the number of changes low (around 4), but when changes do occur, they spread through the whole population.

This, then, is a configuration in which the behaviour of the simulation seems akin to real linguistic change in the desired senses. The community has a clear norm, with 


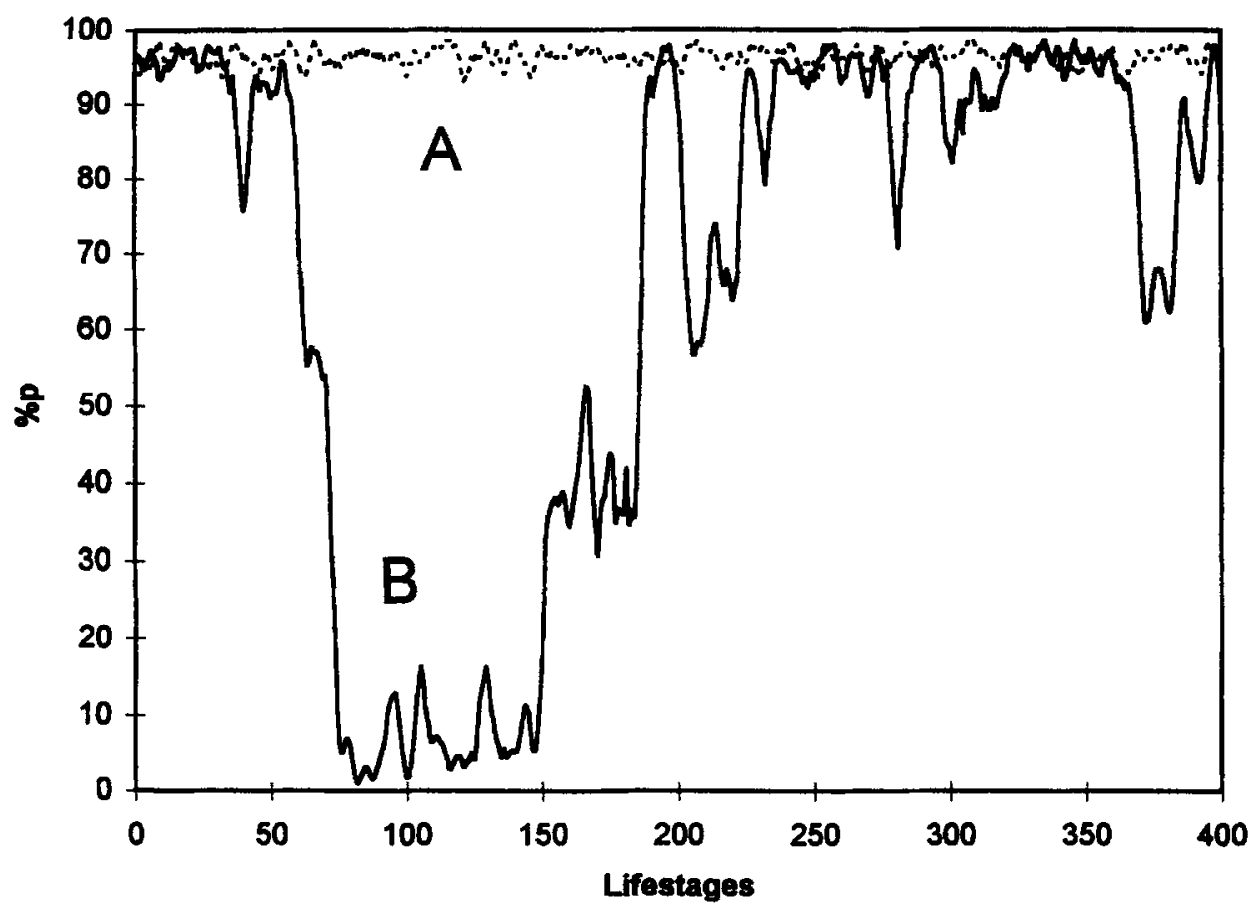

Fig. 6. The percentage of individuals having $p$ over 400 lifestages, with social selection and different population distributions of status (A: Status $(1-20)^{2}$, B: With hyperinfluential individuals).

pockets of variation which usually die away, but occasionally become large enough to pass the threshold and replace the population norm. The community then settles on the new norm for a period of equilibrium before the next change is actuated.

The incorporation of social selection, then, produces an outcome interestingly akin to real life. This supports what is evident from the empirical work of sociolinguistics; that it is because learners attend preferentially to the sources they are most socially attracted to that language change is actuated. The results also suggest that the influence differentials between people may be really quite large. The simulation lacks realism in a crucial respect, however. In the simulation, status and linguistic behaviour are not connected. In life, status is not static but is actively manipulated by people, not least by altering the way they speak.

\section{Functional selection}

The final type of alteration to the simulation we have to consider is that of functional selection. To recapitulate, this is the idea that there are inherent biases against certain linguistic items, for example because they are hard to acquire, which causes languages to change away from them. In this section, we consider the effects of such biases on the dynamics of the simulation. We will investigate how large a bias is 


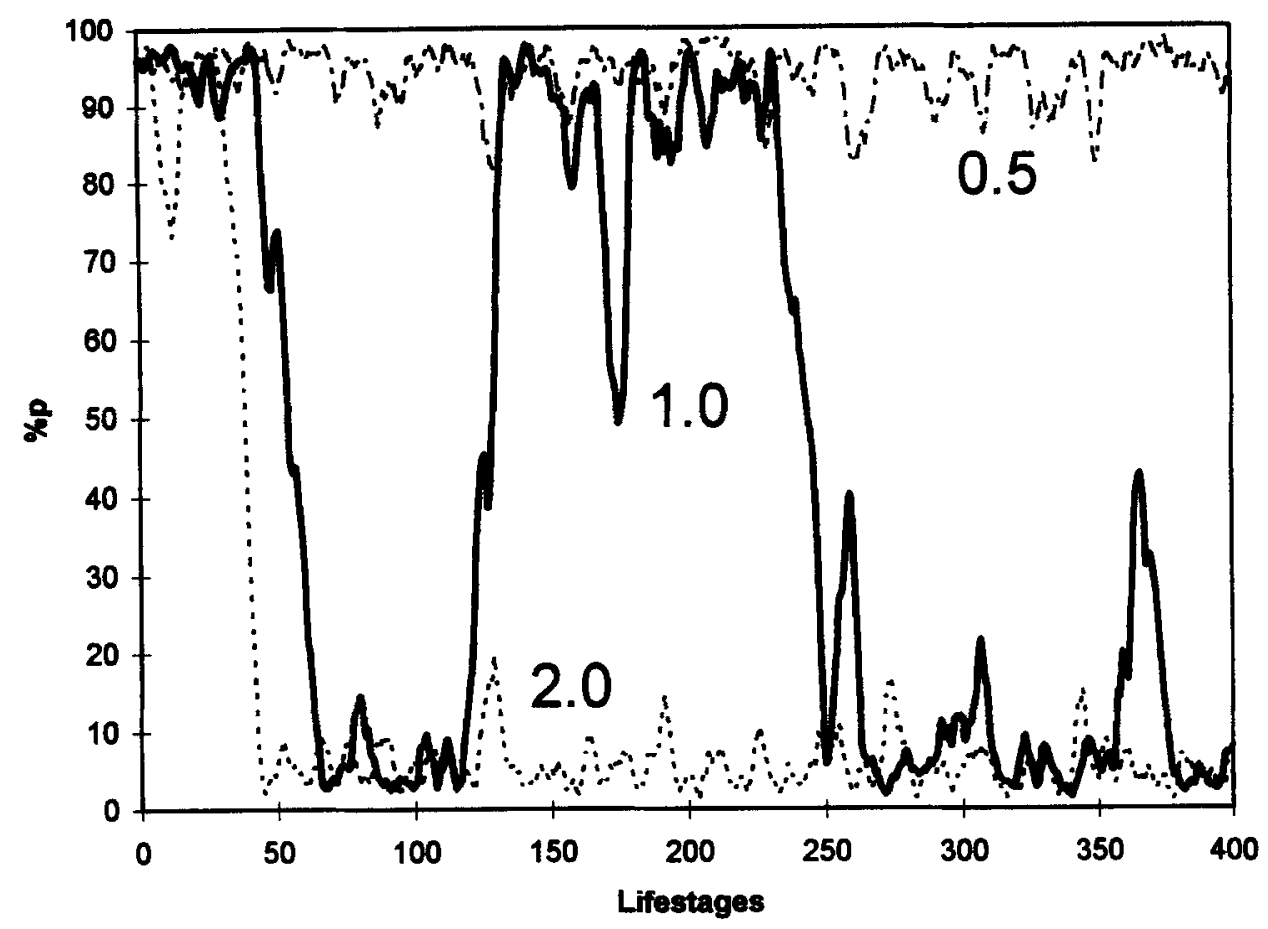

Fig. 7. The percentage of individuals having $p$ over 400 lifestages, with social selection and different biases attaching to $q\left(\mathrm{~b}_{q}=0.5,1\right.$ and 2$)$.

needed to guarantee that a dominant variant is lost, and whether a variant with a bias against it can ever replace one with no such bias.

First let us consider the case where status is distributed as described above, with some hyperinfluential individuals, and there is a bias attaching to the initially rare variant $q$. For simplicity, we take $b_{p}$ the bias attaching to $p$, to be always 1 , and vary just $b_{q}$. Fig. 7 shows percentage of the individuals having $p$ for runs of the simulation with $\mathrm{b}_{q}$ of 0.5 (bias against $q$ ), 1 (no bias) and 2 (bias towards $q$ ). With the bias against $q, q$ is never able to invade the population. With no bias, as before, $p$ and $q$ replace each other in rapid transitions from time to time, with no overall tendency. With the bias against $q, q$ quickly invades and is never subsequently displaced. Thus the language soon finds the optimal configuration and does not subsequently leave it, though whether languages can drift away from optima, particularly when populations and biases are small, is a question which will be considered in a subsequent paper.

We can also ask how large the bias must be in order for the language to stabilise at one variant or the other. Fig. 8 shows that even a very small bias $\left(b_{q}\right.$ of 0.9 or 1.1) ensures a stable and uninvadeable equilibrium of the favoured variant.

We should also consider the case where functional selection is operating but social selection is not. It may be the case that small biases are sufficient to cause lan- 


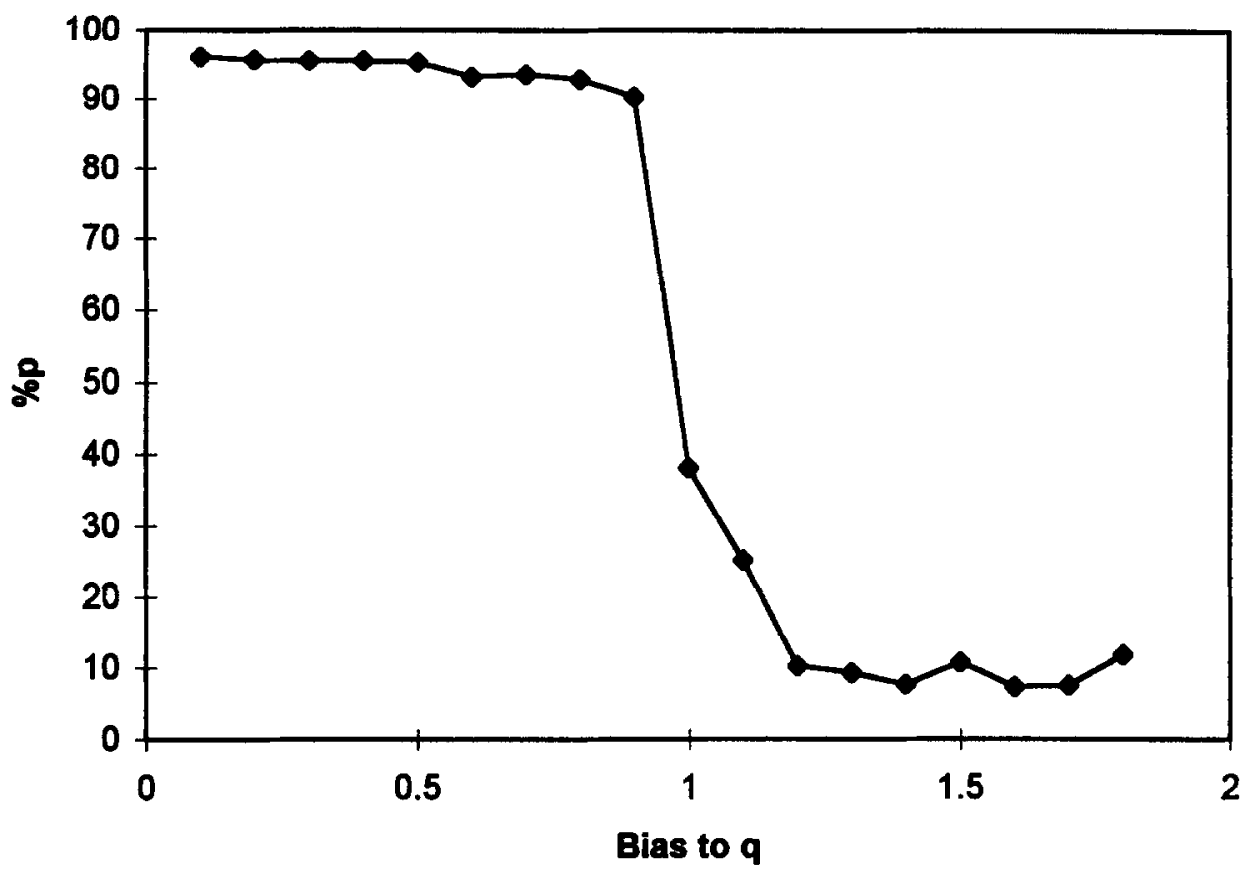

Fig. 8. The mean percentage of individuals having $p$ over 400 lifestages of the simulation with different biases towards or against $q(1=$ no bias $)$.

guage change in the absence of differences in social influence. In fact this does not appear to be the case. As Fig. 9 shows, even a bias to $q$ of 2 is not sufficient to make it invade in a population where there are no status differentials. Thus it would appear that whilst functional biases can affect the direction of language change, they are unlikely to be sufficient of themselves to allow rare variants to overcome the threshold problem and spread unless they are very large indeed. It is more likely that functional biases operate on top of the potential for change furnished by social selection.

\section{Conclusions}

In this paper I have presented a conceptualisation of the process of language evolution in which each learner samples over the language being used in his whole community, weights different sources according to their social status and social distance from him, and derives his own output accordingly.

This conceptualisation throws into sharp relief the threshold problem for language change; that is, if a population is characterised by variant $p$ at some time, then even if a few individuals start using $q, q$ can never spread to fixation, since learners in the next generation will sample across the whole community and adopt $p$ which is hugely more common. Because of the threshold problem, it is hard to see how it is 


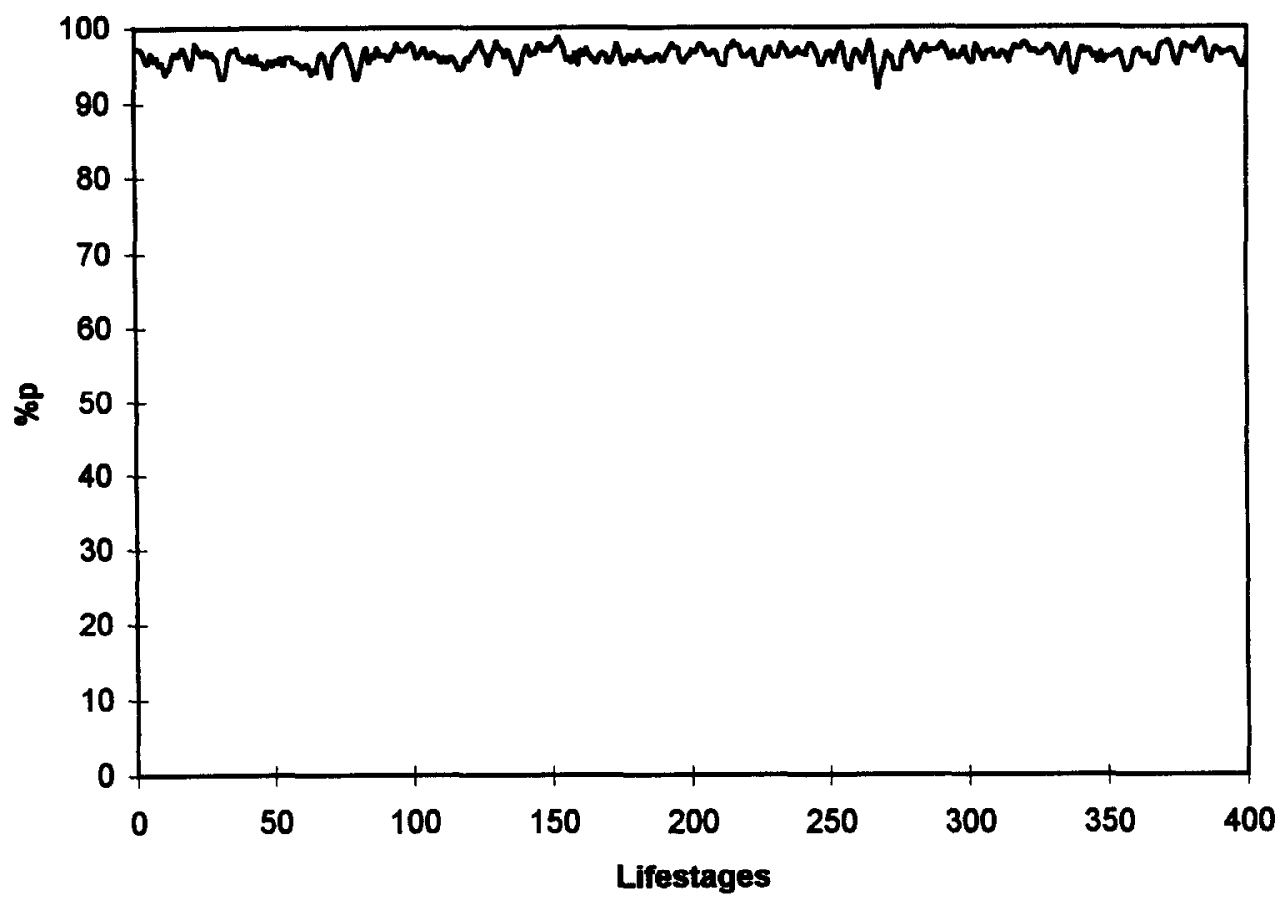

Fig. 9. The percentage of individuals having $p$ over 400 lifestages of the simulation with the bias to $q$ set at 2 but no status differentials.

that new norms sweep periodically through speech communities as we know they do in real life.

The main solution to this problem suggested by the simulations in this paper is that also urged by sociolinguists. Changes are adopted because some speakers are much more influential than others as social models. Their idiosyncrasies thus have a good chance of prevailing against the numerical majority of the community. Indeed, when such differentials are built into the simulation, periods of linguistic stasis and conformity are punctuated with rapid waves during which a new norm sweeps the community.

The simulation also confirmed some basic intuitions about speech communities. Increasing the parochialism of learners (by manipulating the distance parameter) led to a loss of homogeneity and the evolution of separate dialects in the model, as did decreasing the importance learners give to conformity (by decreasing the $a$ parameter).

Finally we have considered the role of functional biases in determining the fate of competing linguistic variants. The simulation suggested that even a small bias was sufficient to determine which of two variants was fixed in a dynamic population. This broadly accords with previous findings using other approaches (e.g. Kirby, 1995), and supports the assumption of typological linguists that differences in fre- 
quency of different language configurations in the world are related to differences in the ease of their acquisition and use (e.g. Tomlin, 1986; Hawkins, 1993). However, it does not seem that functional biases alone can account for the ever changing nature of language. Without the potential for change provided by differences in social influence, functionally favoured variants might never overcome the threshold required to displace prior norms. Functional biases may therefore affect the direction of language change rather than providing sufficient conditions for it to occur. There are probably many linguistic items for which the different variant have absolutely no functional advantage or disadvantage. The incessant change of languages on these items must be a purely social process.

The Social Impact model as set up here can be used to address many further questions such as whether changing the internal structure, demography or size of a speech community affects the rate of linguistic change or the size of bias needed for one variant to replace another. I will address some of these issues in a subsequent paper. How persuasive the reader finds that paper will depend upon how much faith she has in the conceptual and computational framework used here. Clearly, it has been simplistic. We have considered just two competing items instead of the huge set of ambiguous, structurally-related items which confront the real learner. We have made the speaker's competence monolithic, rather than allowing speakers to have access to different dialects or registers. We have fixed social status for life rather than allowing it to be a dynamic variable which depends, inter alia, on language use.

Though these and other limitations should be borne in mind, they are not necessarily reasons for ignoring the arguments and implications presented here. All theory-building involves simplification and idealisation. In writing a computer simulation one has to be painfully precise about what one's simplifications and assumptions are, and the simulation at least tests whether the imagined consequences do really follow from a given process. A simulation, however simple, is thus a huge advance on the purely verbal arguments which are more traditionally employed in the human sciences. The results shown here should thus be accorded some significance in the search for a theory of language change.

\section{References}

Aitchison, J., 1991. Language change: Progress or decay? 2nd edition. Cambridge: Cambridge University Press.

Andersen, H., 1978. Perceptual and conceptual factors in abductive change. In: J. Fisiak (ed.), Recent developments in historical phonology, 1-23. The Hague: Mouton.

Andersen, H., 1988. Center and periphery: Adoption, diffusion and spread. In: J. Fisiak (ed.), Historical dialectology: Regional and social, 39-84. The Hague: Mouton.

Bikhchandani, S., D. Hirshleifer and I. Welch, 1998. Learning from the behavior of others: Conformity, fads, and informational cascades. Journal of Economic Perspectives 12(3), 151-170.

Boyd, R. and P.J. Richerson, 1985. Culture and the evolutionary process. Chicago, IL: University of Chicago Press.

Cavalli-Sforza, L.L. and M.W. Feldman, 1981. Cultural transmission and evolution: A quantitative approach. Princeton, NJ: Princeton University Press.

Chambers, J.K., 1995, Sociolinguistic theory. Oxford: Blackwell. 
Cutler, A., J.A. Hawkins and G. Gilligan, 1985. The suffixing preference: A processing explanation. Linguistics $23,723-750$.

Hägerstrand, T., 1965. A Monte Carlo approach to diffusion. European Journal of Sociology 6, 43-67. Hawkins, J.A., 1994. A performance theory of order and constituency. Cambridge: Cambridge University Press.

Hudson, R.A., 1996. Sociolinguistics. 2nd edition. Cambridge: Cambridge University Press.

Hurford, J.R., 1991. The evolution of the critical period for language acquisition. Cognition 40, 159201.

Jackson, J.M. and B. Latané, 1983. All alone in front of all those people: Stage fright as a function of number and type of co-performers. Journal of Personality and Social Psychology 40, 73-85.

Keller, R., 1994. On language change: The invisible hand in language. London: Routledge.

Kimura, M., 1983. The neutral theory of molecular evolution. Cambridge: Cambridge University Press.

King, R.D., 1969. Historical linguistics and generative grammar. Englewood Cliffs, NJ: Prentice Hall.

Kirby, S., 1993. Adaptive explanations for language universals: A model of Hawkins' performance theory. Sprachtypologie und Universalienforschung 47, 186-210.

Kirby, S., 1995. Competing motivations and emergence: Explaining implicational hierarchies. Edinburgh Occasional Papers in Linguistics, 1.

Kirby, S., 1999. Function, selection, and innateness - The emergence of language universals. Oxford: Oxford University Press.

Labov, W., 1963. The social motivation of a sound change. Word 19, 273-309.

Latané, B., 1981. The psychology of social impact. American Psychologist 36, 343-365.

Latané, B. and S. Nida, 1981. Ten years of research on group size and helping. Psychologial Bulletin 89, 308-324.

Latané, B. and S. Wolf, 1981. The social impact of majorities and minorities. Psychological Review 88, $438-453$.

LePage, R.B., 1968. Problems of description in multilingual communities. Transactions of the Philological Society 1968, 189-212.

Lindblom, B., S. Guion, S. Hura, S-J. Moon and R. Willerman, 1995. Is sound change adaptive? Revista di Linguistica 7, 5-37.

Lütdke, H., 1986. Esquisse d'une théorie du changement langagier. La Linguistique 22, 3-46.

Milroy, L., 1980. Language and social networks. Oxford: Blackwell.

Nettle, D., 1996. The evolution of linguistic diversity. Ph.D. dissertation, University of London.

Nettle, D., 1999. Linguistic diversity. Oxford: Oxford University Press.

Nowak, A., J. Szamrej and B. Latané, 1990. From private attitude to public opinion: A dynamical theory of social impact. Psychological Review 97. 362-376.

Sapir, E., 1921/1970. Language. London: Harcourt, Brace and World.

Tomlin, R., 1986. Basic constituent orders: Functional principles. London: Croom Helm.

Wolff, E.N., 1998. Recent trends in the size distribution of household wealth. Journal of Economic Perspectives 12(3), 131-150. 